\title{
Continuous Sliding Mode Tracking Control with Robust Observer for Nonlinear Systems with Control Gain Uncertainty ${ }^{*}$
}

\author{
Xiaoyu Zhang \\ School of Electronic and Information Engineering, North China Institute of Science \\ and Technology, Beijing 065201, China \\ ysuzxy@aliyun.com
}

\begin{abstract}
Chattering is one of the main shortcomings of sliding mode control. In this paper method using observer to designing continuous sliding mode control (CSMC) law for a class of nonlinear uncertain systems with uncertainty in control channel is addressed. By constructing a robust observer based on LMI method, variable structure control (VSC) law can be designed on the derivative of the control signal. Consequently chattering phenomenon is eliminated. Simulation results on a numerical example are reported to illustrate the system performance and the feasibility of the control algorithm.
\end{abstract}

Keywords: Sliding mode control, observer, nonlinear systems, robust control

\section{Introduction}

Many research results about the chattering problem of variable structure systems (VSS) were reported recently. Among them, high order sliding mode control (HOSMC) is a good approach to form continuous sliding mode control (CSMC) and certainly avoiding chattering [1-4]. In this method, not only the sliding mode but its $n-1$ order successive derivatives are stabilized to zero which will be called $n$ order sliding mode.

As a special case of HOSMC, second order sliding mode control was presented by G.Bartolini and P. Pydynowski [5-9], in which a continuous first-order estimator is acquired to replace the nonlinear dynamic function in the sliding mode firstly and then a second-order auxiliary system is obtained to design the time derivative of the control signal. Nevertheless, some differential inequalities with several conditions are required to be solved, which introduce difficulties for control design. Afterwards, the method was mended and the results were improved [6]. Sub-optimal variable structure control law is obtained based on the proposed results [7-8], which conduces to better performance of closed-loop systems. The same research about multi-input systems is discussed in [9].

In succession, HOSMC with observer was also been investigated [10-13]. It is used to resolve issues when system state variables cannot be measured directly. For example, Shtessel, propose HOSM Observer for a Class of Nonlinear MIMO Systems to deal with the immeasurable state feedback problem [14], Bejarano et al. realize HOSMC by Min-Max optimization based on integral sliding mode [15]. However, the HOSMC method or sub-

\footnotetext{
* This work is supported by the National Natural Science Foundation of China (61304024), the Fundamental Research Funds for the Central Universities (3142013055), the Natural Science Foundation of Hebei Province (F2013508110) and the Science and Technology plan projects of Hebei Provincial Education Department (Z2012089).
} 
optimal VSC theory has some shortcomings. For example, the differential inequalities with constraints are required to be resolved [6], the search program of sub-optimal control signal is very complicated and some conditions are required. Additionally, the high order derivatives of sliding mode are necessary to be estimated by differentiators [1-4].

In this paper, utilization of observer to realize HOSMC is considered. A method to design continuous SMC (second order SMC) based on a robust observer is presented to eliminate the chattering phenomenon of VSC system. It has many merits such as:

(1) The design of the observer is via LMI technique and hence possesses convenience.

(2) The VSC control is designed on the derivative of the control signal and independent on the observer design.

Firstly we selected a suitable sliding mode and then a second-order robust observer was constructed via LMI method inspired by [16-20]. A general sliding mode control law was designed on the time derivative of the control signal, and then the continuous sliding mode control law was obtained. Simulation research validated the CSMC design via LMI method.

This paper is organized as follows. In Section II, the problem formulation is provided. The constructing of the second-order robust observer is presented in Section III as a theorem. The designing of the derivative of the control input is generalized in Section IV. The simulation results on a numerical example are shown in Section V. Finally, Section VI gives the conclusions.

\section{Problem Formulation}

Consider a class of uncertain nonlinear system with high-order dynamics as follows,

$$
\begin{aligned}
& \dot{x}_{i}=x_{i+1}, \quad i=1,2, \cdots, n-1 \\
& \dot{x}_{n}=f(x, t)+(b+\Delta b(t)) u(t)
\end{aligned}
$$

where $x=\left[\begin{array}{lll}x_{1} & \cdots & x_{n}\end{array}\right]^{T} \in \mathfrak{R}^{n}$ is the state vector and can be measured, $f(x, t) \in \mathfrak{R}$ is the unknown nonlinear dynamic, $b \in \mathfrak{R}$ is the nonlinear gain, $\Delta b(t) \in \mathfrak{R}$ is its uncertainty, $u$ is the single control signal.

Suppose that the desired trajectory is $r(t)$ and produced by the following tracked model

$$
\begin{aligned}
& \dot{x}_{d i}=x_{d(i+1)}, \quad i=1,2, \cdots, n-1 \\
& \dot{x}_{d n}=-\sum_{j=1}^{n} a_{j-1} x_{d j}+r(t)
\end{aligned}
$$

with its state vector $x_{d}=\left[\begin{array}{lll}x_{d 1} & \cdots & x_{d n}\end{array}\right]^{T}$ and parameters $a_{j-1}, j=1,2, \cdots, n$ coefficients of a Hurwitz polynomial.

To ensure the effectiveness of our method, the following assumptions for the systems (1), (2) and a lemma of matrices inequality are introduced.

Assumption 1. The nonlinear uncertainty $f$ has one-order continuous time derivate and satisfies that $\left|\frac{d f}{d t}-\hat{f}_{d}\right| \leq \zeta$ with $\zeta$ is positive scalar and $\hat{f}_{d}$ is the estimation of $\frac{d f}{d t}$.

Assumption 2. The time derivate of the trajectory $r(t)$ and other tracked signal can be acquired. 
Assumption 3. The control gain uncertainty $\Delta b(t)$ and its time derivative are all bound, namely $\xi_{1} \leq|\Delta b(t)| \leq \xi_{2},|\Delta \dot{b}(t)| \leq \mu$ with $\xi_{1}, \xi_{2}, \mu$ are positive scalars.

Assumption 4. The control gain uncertainty $\Delta b(t)$ and the time derivative of control gain uncertainty $\Delta \dot{b}(t)$ both satisfy

$$
\Delta b(t)=H_{0} F_{0}(t) E_{0}, \Delta \dot{b}(t)=H_{1} F_{1}(t) E_{1},
$$

where $H_{0}, H_{1}, E_{0}, E_{1}$ are all constant matrices with suitable dimension, $F_{0}(t)$ and $F_{1}(t)$ are Lebesgue-measurable and fulfill $F_{i}^{T}(t) F_{i}(t) \leq I(i=0,1)$ with $I$ is identity matrix.

Lemma $1^{[21]}$. Suppose matrix $F(t)$ satisfies $F^{T}(t) F(t) \leq I$ with $I$ is identity matrix, then for any constant scalar $\varepsilon>0$ and real constant matrices $D, E$ with suitable dimensions, the following inequality

$$
D F(t) E+E^{T} F^{T}(t) D^{T} \leq \varepsilon D D^{T}+\varepsilon^{-1} E^{T} E
$$

is valid.

Consider the augmented system

$$
\begin{aligned}
\dot{x}_{i} & =x_{i+1}, \quad i=1,2, \cdots, n \\
\dot{x}_{n+1} & =\frac{d f}{d t}+b \dot{u}(t)+\Delta b(t) \dot{u}(t)+\Delta \dot{b}(t) u(t)
\end{aligned}
$$

and the augmented model

$$
\begin{aligned}
\dot{x}_{d i} & =x_{d(i+1)}, \quad i=1,2, \cdots, n \\
\dot{x}_{d(n+1)} & =-\sum_{j=1}^{n} a_{j-1} x_{d(j+1)}+\dot{r}(t)
\end{aligned}
$$

where

$$
\begin{gathered}
x_{n+1}=f(x, t)+(b+\Delta b(t)) u(t), \\
x_{d(n+1)}=-\sum_{j=1}^{n} a_{j-1} x_{d j}+r(t)
\end{gathered}
$$

are the corresponding augmented state variables.

The control problem is to construct a control law $u(x, s, t)$ such that the error vector

$$
e=\left[\begin{array}{lll}
e_{1} & \cdots & e_{n+1}
\end{array}\right]^{T}, e_{i}=x_{d i}-x_{i}, \quad i=1,2, \cdots, n+1
$$

converges to the tolerance range, where $s$ is the sliding mode surface

$$
s=c^{T} e, \quad c=\left[\begin{array}{lllll}
c_{1} & \cdots & c_{n-1} & c_{n} & 1
\end{array}\right]^{T},
$$

and the vector $c$ makes a Hurwitz polynomial.

Once the system (1) stepped into the sliding mode $s=0$, the closed-loop system will become a $n$-order linear system. Furthermore, the $n$-order linear system is stable as the sliding mode coefficient $c$ is Hurwitz. However, $x_{n+1}$ and $s$ are immeasurable in the system (1), which will introduce difficulty for control design. In the following section, we will design robust observer to approximate them. 


\section{Auxiliary Robust Observer}

The time derivate of the sliding mode $s$ can be described as follows from (6),

where

$$
\begin{aligned}
\dot{s}(t) & =\dot{e}_{n+1}+\sum_{i=1}^{n} c_{i} \dot{e}_{i}=\dot{x}_{d(n+1)}-\dot{x}_{n+1}+c_{n} e_{n+1}+\sum_{i=1}^{n-1} c_{i} e_{i+1} \\
& =-\dot{x}_{n+1}-c_{n} x_{n+1}+\dot{x}_{d(n+1)}+c_{n} x_{d(n+1)}+\sum_{i=1}^{n-1} c_{i} e_{i+1} \\
& =-\dot{x}_{n+1}-c_{n} x_{n+1}+\varphi
\end{aligned}
$$

$$
\varphi=\dot{x}_{d(n+1)}+c_{n} x_{d(n+1)}+\sum_{i=1}^{n-1} c_{i} e_{i+1} .
$$

Denote $v=b \dot{u}(t)$ and by using (3) and (8), the following can be achieved,

$$
\begin{gathered}
\dot{x}_{n+1}=\frac{d f}{d t}+v+\Delta b(t) \dot{u}(t)+\Delta \dot{b}(t) u(t), \\
\dot{s}=-\frac{d f}{d t}-c_{n} x_{n+1}-v-\Delta b(t) \dot{u}(t)-\Delta \dot{b}(t) u(t)+\varphi .
\end{gathered}
$$

According to the equations (8), (9), $\varphi$ is measurable and $v$ is the control derivative to be designed. Define the output $y_{1}$ of the auxiliary system (9) as follows,

$$
y_{1}=x_{d(n+1)}+\sum_{i=1}^{n} c_{i} e_{i} .
$$

Using the equations (9) and (10) yields the following,

where

$$
\begin{aligned}
{\left[\begin{array}{c}
\dot{x}_{n+1} \\
\dot{s} \\
\dot{u} \\
\ddot{u}
\end{array}\right] } & =\left(A_{0}+\Delta A_{0}\right)\left[\begin{array}{c}
x_{n+1} \\
s \\
u \\
\dot{u}
\end{array}\right]+\left[\begin{array}{l}
0 \\
1 \\
0 \\
0
\end{array}\right] \varphi+B_{0} \dot{v}+B_{1} \frac{d f}{d t}, \\
y & =C^{T}\left[\begin{array}{llll}
x_{n+1} & s & u & \dot{u}
\end{array}\right]^{T},
\end{aligned}
$$

$$
\begin{gathered}
A_{0}=\left[\begin{array}{cccc}
0 & 0 & 0 & b \\
-c_{n} & 0 & 0 & -b \\
0 & 0 & 0 & 1 \\
0 & 0 & 0 & 0
\end{array}\right], B_{0}=\left[\begin{array}{c}
0 \\
0 \\
0 \\
b^{-1}
\end{array}\right], C=\left[\begin{array}{cccc}
1 & 1 & 0 & 0 \\
0 & 0 & 1 & 0 \\
0 & 0 & 0 & 1
\end{array}\right]^{T}, \\
B_{1}=\left[\begin{array}{c}
1 \\
-1 \\
0 \\
0
\end{array}\right], \Delta A_{0}=\left[\begin{array}{cccc}
0 & 0 & \Delta \dot{b}(t) & \Delta b(t) \\
0 & 0 & -\Delta \dot{b}(t) & -\Delta b(t) \\
0 & 0 & 0 & 0 \\
0 & 0 & 0 & 0
\end{array}\right] .
\end{gathered}
$$

The variables $x_{n+1}$ and $s$ are necessary for resolving the control problem. However, they are all immeasurable.

Consider the following observer,

$$
\begin{aligned}
{\left[\begin{array}{c}
\dot{\bar{x}}_{n+1} \\
\dot{\bar{s}}
\end{array}\right] } & =\left[\begin{array}{cc}
0 & 0 \\
-c_{n} & 0
\end{array}\right]\left[\begin{array}{c}
\bar{x}_{n+1} \\
\bar{s}
\end{array}\right]+\left[\begin{array}{c}
0 \\
1
\end{array}\right] \varphi+\left[\begin{array}{c}
1 \\
-1
\end{array}\right] v+\left[\begin{array}{c}
1 \\
-1
\end{array}\right] \hat{f}_{d}+v, \\
\bar{y} & =C^{T}\left[\begin{array}{llll}
\bar{x}_{n+1} & \bar{s} & u & \dot{u}
\end{array}\right]^{T},
\end{aligned}
$$

where $v$ is a new control input for the observer and to be designed. The variables $\bar{x}_{n+1}$ and $\bar{s}$ 
are the observation value of $x_{n+1}, s$ respectively.

Define the control input $v$ as

$$
v=-G_{1} \tilde{y}
$$

where $G_{1}$ is feedback gain matrix to be determined and

$$
G=\left[\begin{array}{ll}
G_{1}^{T} & G_{2}^{T}
\end{array}\right]^{T}, G \in R^{4 \times 3},
$$

$\tilde{y}$ is the measurable output error. Denote a vector of the state variable as the following,

$$
\bar{e}=\left[\begin{array}{llll}
x_{n+1}-\bar{x}_{n+1} & s-\bar{s} & u & \dot{u}
\end{array}\right]^{T},
$$

which includes observing error $x_{n+1}-\bar{x}_{n+1}$ and $s-\bar{s}$.

According to the equation (11), (12), the observation error equation can be depicted as the following linear system,

$$
\begin{aligned}
\dot{\bar{e}} & =\left(A_{0}+\Delta A_{0}\right) \bar{e}+G \tilde{y}+B_{0} \dot{v}+B_{1} \tilde{f}_{d}, \\
\tilde{y} & =C^{T} \bar{e},
\end{aligned}
$$

where $\tilde{f}_{d}=\frac{d f}{d t}-\hat{f}_{d}$ is considered as immeasurable perturbation.

It can be verified that $\left[A_{0}, C^{T}\right]$ is observable. Therefore the problem becomes to design the control law $v$ via output feedback $\tilde{y}$ to stabilize the error system (15) which state vector $\bar{e}$ contains parts of variables that cannot be measured. Namely, the parameter $G$ and the control $v$ are to be determined so that the observation errors of the variables $x_{n+1}$ and $s$ converge to zero or a tolerance range.

Main result of designing the parameters $G$ of the observer (12) and the control input (13) is given as the following theorem.

Theorem 1. For any small positive scalar $\delta$, if there exist parameter $G \in \mathfrak{R}^{4 \times 3}$, symmetric positive-definite matrices

$$
P=\left[\begin{array}{rr}
P_{1} & 0 \\
0 & 1
\end{array}\right], P_{1}=P_{1}^{T}, P_{1}>0\left(P_{1} \in R^{3 \times 3}\right),
$$

$R \in R^{4 \times 4}, R>0, R=R^{T}$ and some scalar $\varepsilon>0$ satisfying the following LMI,

$$
\left[\begin{array}{cccc}
A_{c}^{T} P+P A_{c}+ & E^{T} & P B_{1} & 0 \\
R+\varepsilon P H H^{T} P^{T} & E^{2} & 0 & 0 \\
E & -\varepsilon I & 0 & 0 \\
B_{1}^{T} P & 0 & -\varepsilon I & 0 \\
0 & 0 & 0 & -\frac{\varepsilon \zeta^{2}}{\delta} I
\end{array}\right] \leq 0,
$$

where $A_{c}=A_{0}-G C^{T}, I$ is identity matrix with suitable dimensions, then the observation error system (15) is robust quadratically stable when the norm of the error vector is greater than $\delta$ and consistently boundary when the norm of the error vector is less than $\delta$.

Proof: Select a Lyapunov function as

$$
V=\bar{e}_{1}^{T} P_{1} \bar{e}_{1},
$$

where $\bar{e}_{1}=\left[\begin{array}{lll}x_{n+1}-\bar{x}_{n+1} & s-\bar{s} & u\end{array}\right]^{T}$. Obviously using the equation (15) the Lyapunov function can be given by

$$
V=\bar{e}^{T} P \bar{e}-b^{-2} v^{2} .
$$

Consequently calculate its derivative along the trajectory of system equation (14) with respect to the time, 


$$
\begin{aligned}
\dot{V} & =\dot{\bar{e}}^{T} P \bar{e}+\bar{e}^{T} P \dot{\bar{e}}-2 b^{-2} v \dot{v} \\
& =\bar{e}^{T}\left(A_{c}^{T} P+P A_{c}+\Delta A_{0}^{T} P+P \Delta A_{0}\right) \bar{e}+2 B_{1}^{T} P \bar{e} \tilde{f}_{d} .
\end{aligned}
$$

By Lemma 1, we have

$$
B_{1}^{T} P \bar{e}\left[\tilde{f}_{d}\right]+\left[\tilde{f}_{d}\right]^{T} \bar{e}^{T} P B_{1} \leq \varepsilon^{-1} \bar{e}^{T} P B_{1} B_{1}^{T} P \bar{e}+\varepsilon\left[\tilde{f}_{d}\right]^{T}\left[\tilde{f}_{d}\right],
$$

where $\varepsilon>0$ is an arbitrary scalar. Additionally according to Assumption 1 , the variable $\tilde{f}_{d}$ satisfies the inequality $\left|\tilde{f}_{d}\right| \leq \zeta$. And cccording to Assumption 3, $\Delta A_{0}=H F(t) E$ where

$$
H=\left[\begin{array}{cc}
H_{0} & H_{1} \\
-H_{0} & -H_{1} \\
0 & 0 \\
0 & 0
\end{array}\right], F(t)=\left[\begin{array}{cc}
F_{0}(t) & 0 \\
0 & F_{1}(t)
\end{array}\right], E=\left[\begin{array}{cccc}
0 & 0 & 0 & E_{0} \\
0 & 0 & E_{1} & 0
\end{array}\right] .
$$

Therefore the following inequality can be given

$$
\triangle A_{0}^{T} P+P \Delta A_{0}=P H F(t) E+E^{T} F^{T}(t) H^{T} P^{T} \leq \varepsilon P H H^{T} P^{T}+\varepsilon^{-1} E^{T} E .
$$

Then, substituting the inequality (18) and (19) into (17) yields

$$
\begin{aligned}
\dot{V} & \leq \bar{e}^{T}\left(A_{c}^{T} P+P A_{c}+\varepsilon P H H^{T} P^{T}+\varepsilon^{-1} E^{T} E+\varepsilon^{-1} P B_{1} B_{1}^{T} P\right) \bar{e}+\varepsilon\left[\tilde{f}_{d}\right]^{T} \tilde{f}_{d} \\
& \leq \bar{e}^{T}\left(A_{c}^{T} P+P A_{c}+\varepsilon P H H^{T} P^{T}+\varepsilon^{-1} E^{T} E+\varepsilon^{-1} P B_{1} B_{1}^{T} P\right) \bar{e}+\varepsilon \zeta^{2} \\
& <\bar{e}^{T}\left(A_{c}^{T} P+P A_{c}+R+\varepsilon P H H^{T} P^{T}+\varepsilon^{-1} E^{T} E+\varepsilon^{-1} P B_{1} B_{1}^{T} P\right) \bar{e}+\varepsilon \zeta^{2} .
\end{aligned}
$$

For $\bar{e}^{T} \bar{e}>\delta^{2}$, we can obtain the following inequality from the inequality (20),

$$
\dot{V}<\bar{e}^{T} M \bar{e},
$$

where

$$
M=A_{c}^{T} P+P A_{c}+R+\varepsilon P H H^{T} P^{T}+\varepsilon^{-1} E^{T} E+\varepsilon^{-1} P B_{1} B_{1}^{T} P+\varepsilon \frac{\zeta^{2}}{\delta^{2}} I
$$

According to the statements in theorem 1, the solution of LMI (16) is equivalent to that of $M<0$. Hence, the observation error system (15) is robust quadratically stable when the error vector $\bar{e}^{T} \bar{e}>\delta^{2}$.

For $\bar{e}^{T} \bar{e} \leq \delta^{2}$, we can't determine the negative of the Lyapunov function derivative (17) because the absolute value of the item $\bar{e}^{T}\left(A_{c}^{T} P+P A_{c}+R+\varepsilon^{-1} P B_{1} B_{1}^{T} P\right) \bar{e}$ will be near zero along with $\bar{e} \rightarrow 0$ in the inequality (20). However, we can draw the conclusion that the error vector $\bar{e}$ converges to $\|\bar{e}\| \leq \delta$.

Remark 1. If we choose $\varepsilon=\alpha \delta / \zeta^{2}$ with $\alpha>0$ is some scalar, then the LMI (16) becomes

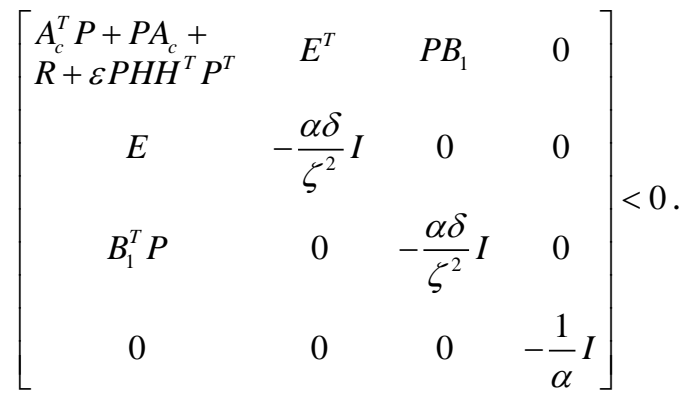

We can directly seek the solution of the LMI (22) $G \in \mathfrak{R}^{4 \times 3}$, symmetric positive-definite matrices $P, R$ and some scalar $\alpha>0$ for any small scalar $\delta$. 
Remark 2. The observation error is independent on the derivative signal $v$. It is significant for control synthesis which allows us design the control derivative $v$ arbitrarily.

\section{Continuous SMC Via the Robust Observer}

Choose the control derivative $v$ as the following,

$$
v=\varphi-c_{n} \bar{x}_{n+1}-\hat{f}_{d}+k \bar{s}+\omega \operatorname{sgn} \bar{s}-\boldsymbol{b}_{2} \tilde{y}
$$

where

$$
\omega=\frac{\xi_{2} b^{-1}\left|\varphi-c_{n} \bar{x}_{n+1}-\hat{f}_{d}-\boldsymbol{b}_{2} \tilde{y}\right|+\mu|u(t)|}{1+\xi_{1} b^{-1}}+\eta
$$

and $k, \eta>0$ are all arbitrary positive scalars, $\boldsymbol{b}_{2}$ is the row vector element of the parameter matrix $G_{1}=\left[\begin{array}{l}\boldsymbol{b}_{1} \\ \boldsymbol{b}_{2}\end{array}\right]$.

Following the above, the control law of the origin system (1) is designed as

$$
u(t)=u(0)+b^{-1} \int_{0}^{t} v(t) d t,
$$

where $u(0)$ is the initial value of the control input $u$. Generally, we can consider $u(0)=0$.

Then the second result can be given by the following theorem.

Theorem 2. The sliding mode observation $\bar{s}$ (defined by observer equation (12)) is asymptotically stable and reaches the origin in finite time by the control (23),(24) and (25).

Proof: By using equation (12), (15), we have

$$
\begin{aligned}
\dot{\bar{s}}= & \bar{s}\left[-(b+\Delta b(t)) \dot{u}(t)-\Delta \dot{b}(t) u(t)-\hat{f}_{d}-c_{n} \bar{x}_{n+1}+\varphi-\boldsymbol{b}_{2} \tilde{y}\right] \\
= & \bar{s}\left[-\left(1+\Delta b(t) b^{-1}\right) v-\Delta \dot{b}(t) u(t)-\hat{f}_{d}-c_{n} \bar{x}_{n+1}+\varphi-\boldsymbol{b}_{2} \tilde{y}\right] \\
= & -\Delta b(t) b^{-1} \bar{s} v-\Delta \dot{b}(t) u(t)-k \bar{s}^{2}-\omega \bar{s} \operatorname{sgn} \bar{s} \\
= & -\Delta b(t) b^{-1} \bar{s}\left[\varphi-c_{n} \bar{x}_{n+1}-\hat{f}_{d}-\boldsymbol{b}_{2} \tilde{y}\right]-\Delta \dot{b}(t) u(t)- \\
& k\left(1+\Delta b(t) b^{-1}\right) \bar{s}^{2}-\omega\left(1+\Delta b(t) b^{-1}\right) \bar{s} \operatorname{sgn} \bar{s} .
\end{aligned}
$$

Afterwards the following inequality can be given by using the inequality (24),

$$
\dot{\bar{s}} \leq-k \bar{s}^{2}-\eta \bar{s} \operatorname{sgn} \bar{s} \text {, }
$$

which shows that the sliding mode observation $\bar{s}$ reaches to the origin in finite time. This convergence character is determined by the arbitrary scalars $k$ and $\eta$. According to the Theorem 1 and its remarks, the observation error is independent on the control derivative $v$.

Consequently, the sliding mode $s$ of the system (1) is boundary stable and satisfies $|s|<\delta\|c\|$ with $\delta$ any small positive scalar. The tracking error vector $e$ converges to zero adjacence along the sliding surface $\bar{s}=0$.

In fact, if we design the control input $v$ as (23), the robust observer (12) becomes

$$
\left[\begin{array}{c}
\dot{\bar{x}}_{n+1} \\
\dot{\bar{s}}
\end{array}\right]=\left[\begin{array}{cc}
0 & 0 \\
-c_{n} & 0
\end{array}\right]\left[\begin{array}{c}
\bar{x}_{n+1} \\
\bar{s}
\end{array}\right]+\left[\begin{array}{c}
0 \\
1
\end{array}\right] \varphi+\left[\begin{array}{c}
1 \\
-1
\end{array}\right] v+\left[\begin{array}{c}
1 \\
-1
\end{array}\right] \hat{f}_{d}-G_{1} \tilde{y} .
$$

The obtained control algorithm is as follows,

1) Design sliding mode parameter $c \in \Re^{n+1}$,

2) Design parameters $G_{1}$ by Theorem 1,

3) Select parameters $k$ and $\omega$ for reaching law (26),

4) Calculate the observation value $\bar{x}_{n+1}$ and $\bar{s}$ by (27), 
5) Calculate the control derivative $v$ by (23),

6) Calculate the control input $u$ by (25).

\section{Numerical Example}

Consider a nonlinear system with three-order dynamic,

$$
\begin{aligned}
{\left[\begin{array}{l}
x_{1} \\
x_{2} \\
x_{3}
\end{array}\right] } & =\left[\begin{array}{l}
x_{2} \\
x_{3} \\
f
\end{array}\right]+\left[\begin{array}{c}
0 \\
0 \\
1+0.5 \sin 10 \pi t
\end{array}\right] u+\left[\begin{array}{c}
0 \\
0 \\
12 \sin 100 \pi t
\end{array}\right], \\
y & =x_{1},
\end{aligned}
$$

where $x=\left[\begin{array}{lll}x_{1} & x_{2} & x_{3}\end{array}\right]^{T}$ is the system state vector, $u$ is the control signal and can be designed by the method in this paper, $y$ is the system output, $f=0.3 e^{0.1 t}+1.2 \cos x_{1}$ is the unknown nonlinear uncertainty, $12 \sin 100 \pi t$ is the matched disturbance.

The tracked model is described as the following,

$$
\begin{aligned}
\dot{x}_{d} & =A_{d} x_{d}+B_{d} r(t), \\
y & =C_{d}^{T} x_{d},
\end{aligned}
$$

where

$$
A_{d}=\left[\begin{array}{ccc}
0 & 1 & 0 \\
0 & 0 & 1 \\
-400 & -170 & -23
\end{array}\right], B_{d}=\left[\begin{array}{c}
0 \\
0 \\
400
\end{array}\right], \quad C_{d}^{T}=\left[\begin{array}{lll}
1 & 0 & 0
\end{array}\right]^{T}
$$

and $x_{d}=\left[\begin{array}{lll}x_{d 1} & x_{d 2} & x_{d 3}\end{array}\right]^{T}$ is the state vector, $r(t)$ is the reference signal.

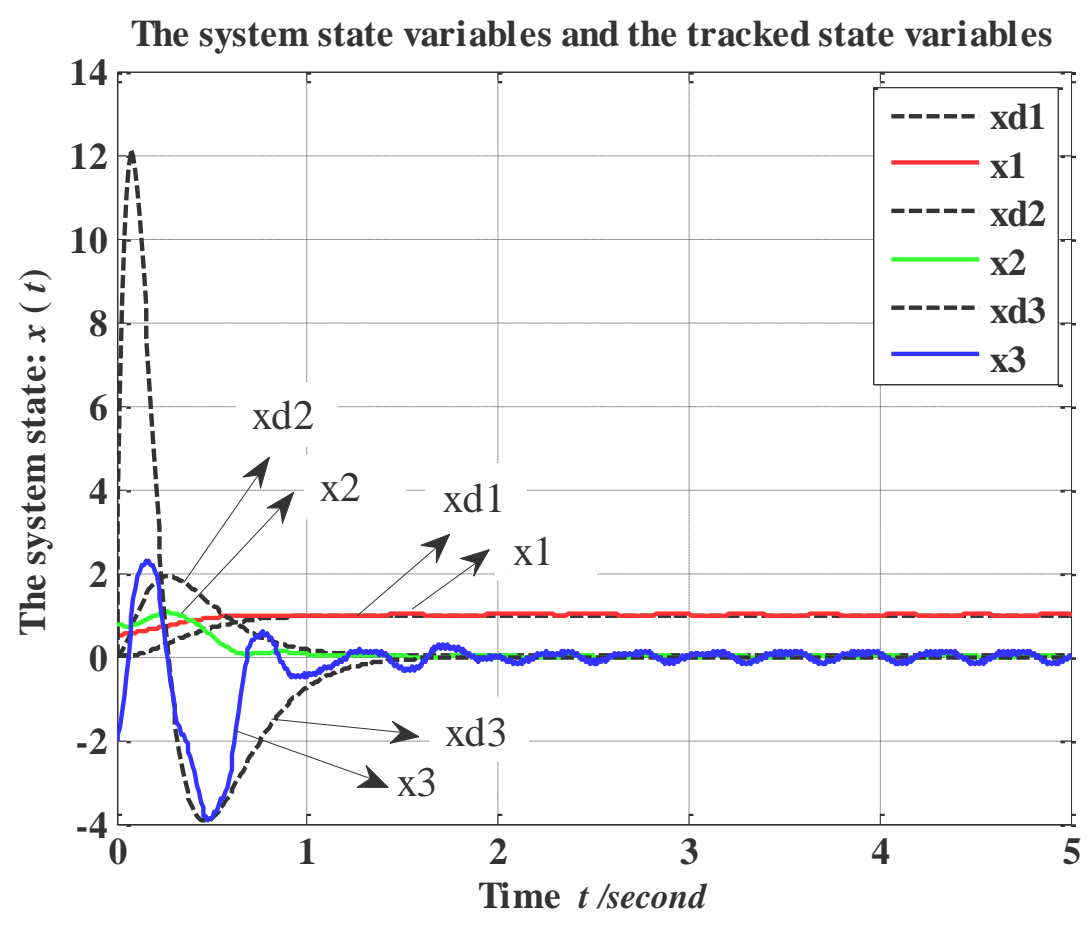

Figure 1. The system out response when the reference signal is step 


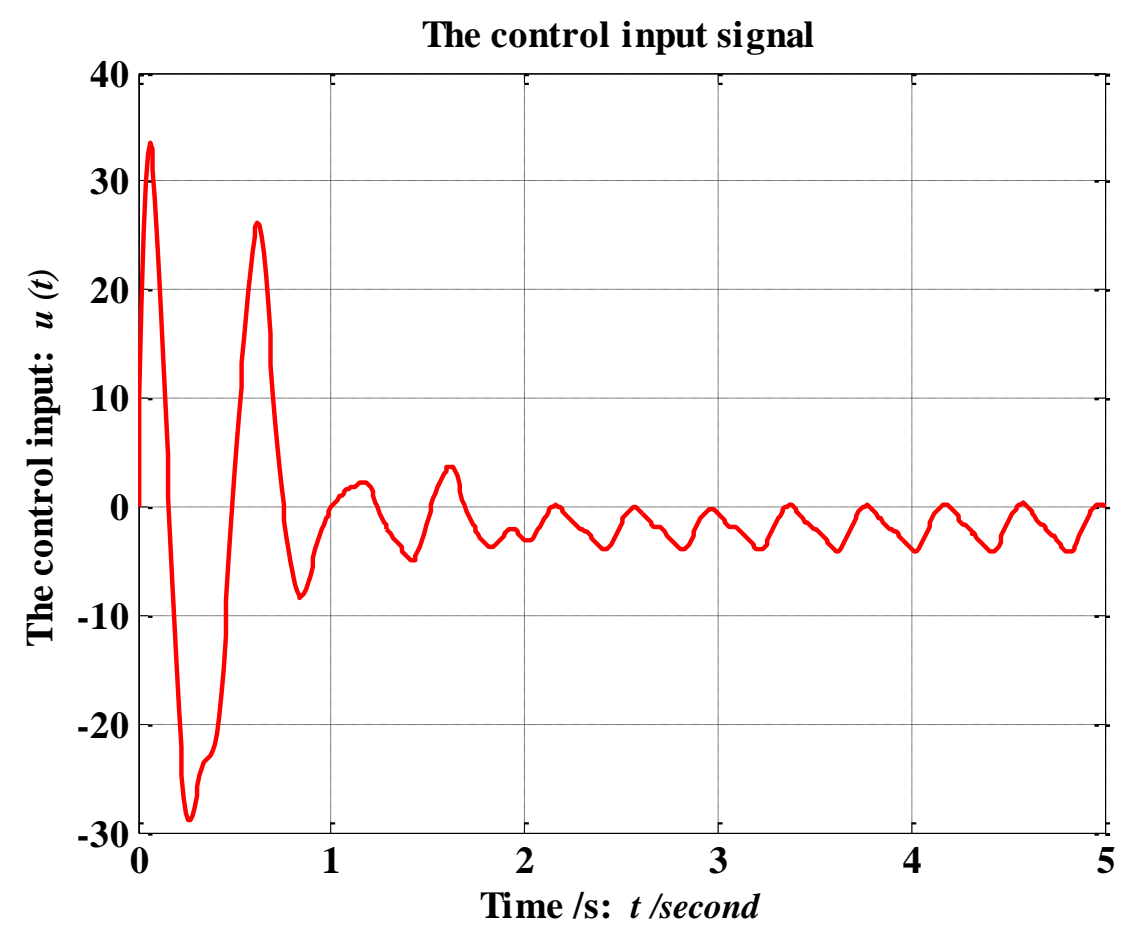

Figure 2. The control input $u$ when the reference signal is step

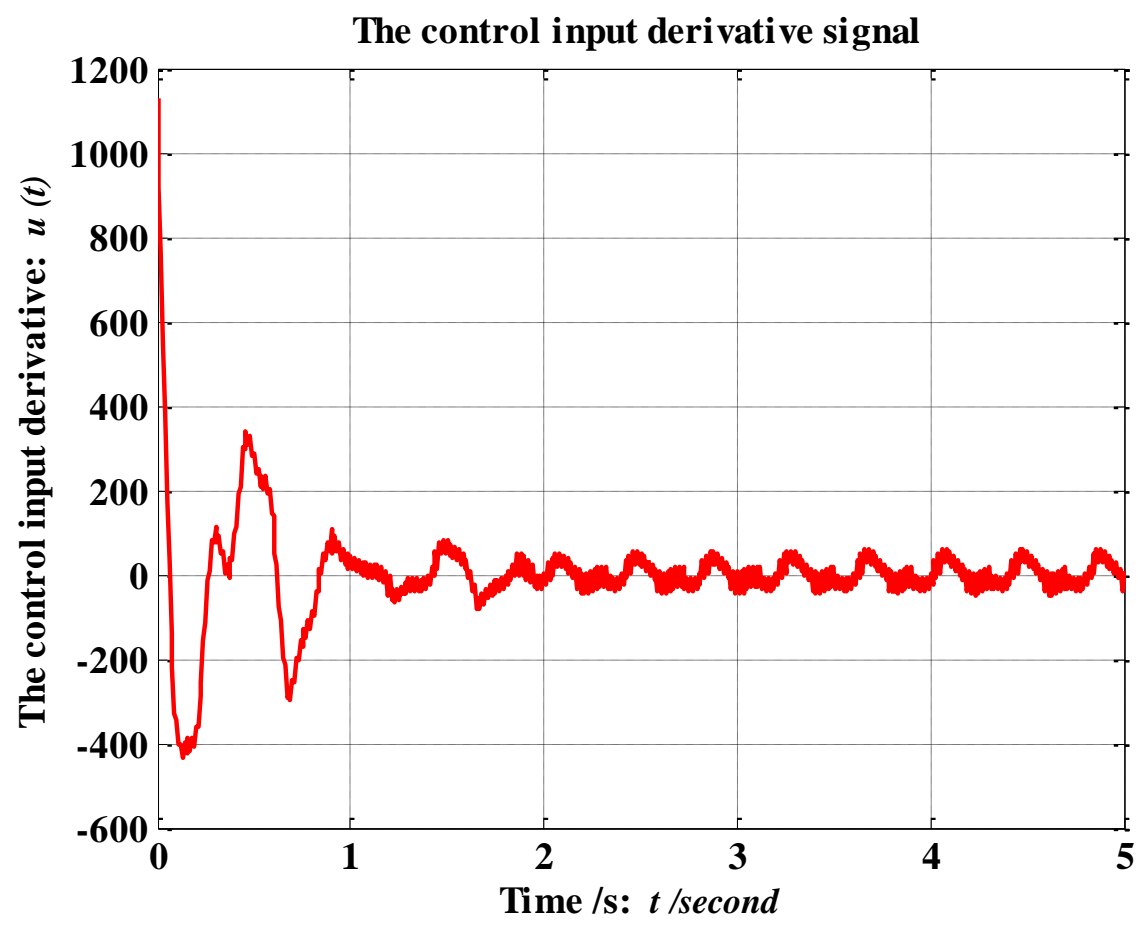

Figure 3. The control derivative signal when the reference is step 
When the reference signal $r(t)=1$, and the system initial states $x=\left[\begin{array}{lll}0 & 0 & 0.5\end{array}\right]^{T}$, the sliding mode parameter is designed as $c=\left[\begin{array}{llll}240 & 180 & 40 & 1\end{array}\right]^{T}$ and the parameters of robust observer $G_{1}=\left[\begin{array}{ccc}0 & 0 & 0 \\ 16 & 0 & 0\end{array}\right]$. Suppose the estimation of the time derivative as $\hat{f}_{d}=0$. Choose the parameters of control derivative $k=4$ and

$$
\omega=0.01+\frac{\left|\varphi-c_{n} \bar{x}_{n+1}-\hat{f}_{d}-b_{2} \tilde{y}\right|+|u(t)|}{3}
$$

by the equaton (24). The output response of the closed-loop system, the control input $u$ and the variation of $v$ are shown in Figure 1-Figure 3 respectively.

Note that the control input $u$ in Figure 2 doesn't converge to zero, because that the uncertainty includes the item $0.3 e^{0.1 t}$ which will increase all along with $t \rightarrow \infty$. It is inevitable to require greater control signal with $t$ increases.

When the reference signal $r(t)$ is rectangle pulse, select different values of the parameters. It is shown that the parameter $G$ has important effects on the system response. $G$ determines the system damp, and mainly make effects on the oscillating actions and the response speed of the system. The corresponding response results are shown in Figure 4 and Figure 5.

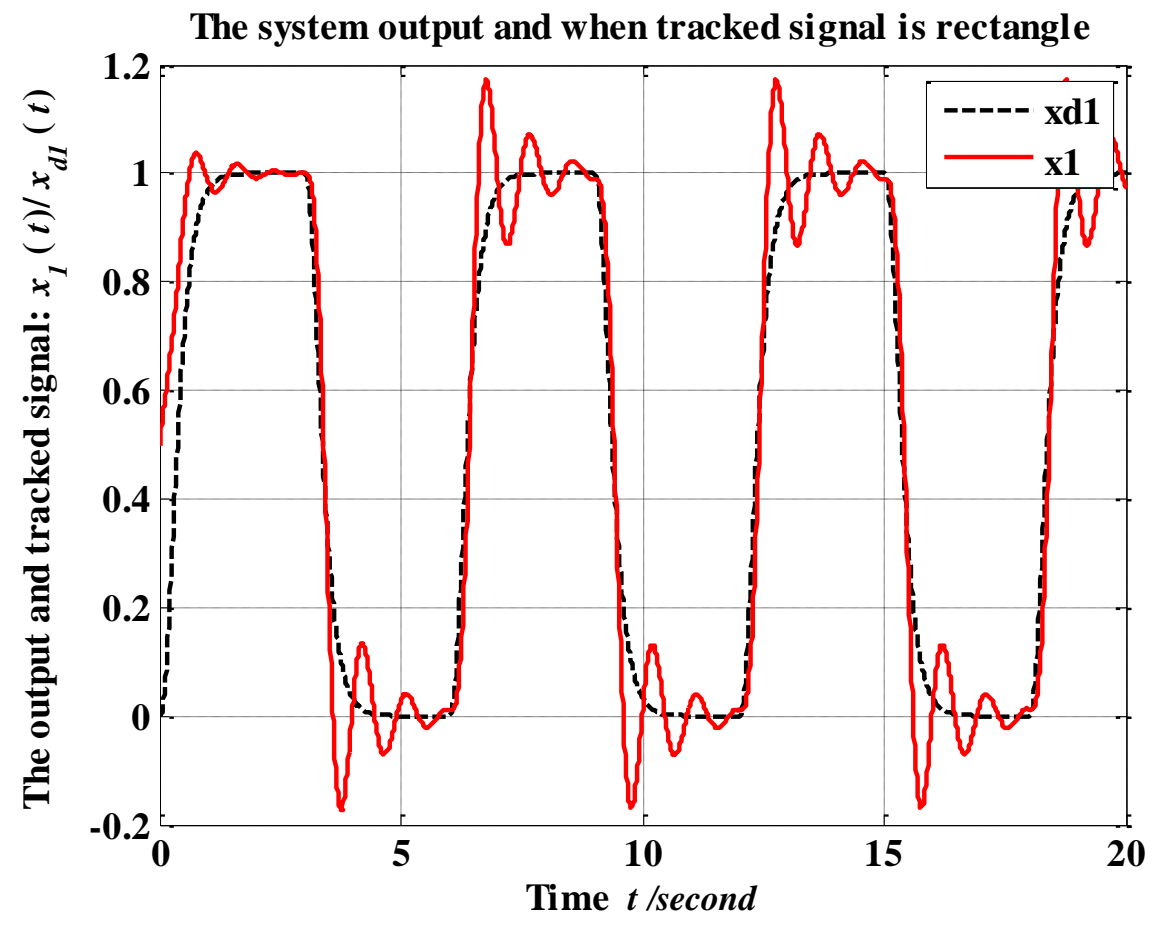

Figure 4. The system out response when the reference signal is rectangle pulse. The parameter of robust observer $\boldsymbol{b}_{2}=\left[\begin{array}{lll}4 & 0 & 0\end{array}\right]$

From the numerical simulation results in Figure 2, it can be seen that the control input $u$ is continuous. The chattering phenomenon of the control input is eliminated by the integrator, because the variable structure control is designed on the derivative signal of the control input $u$. 


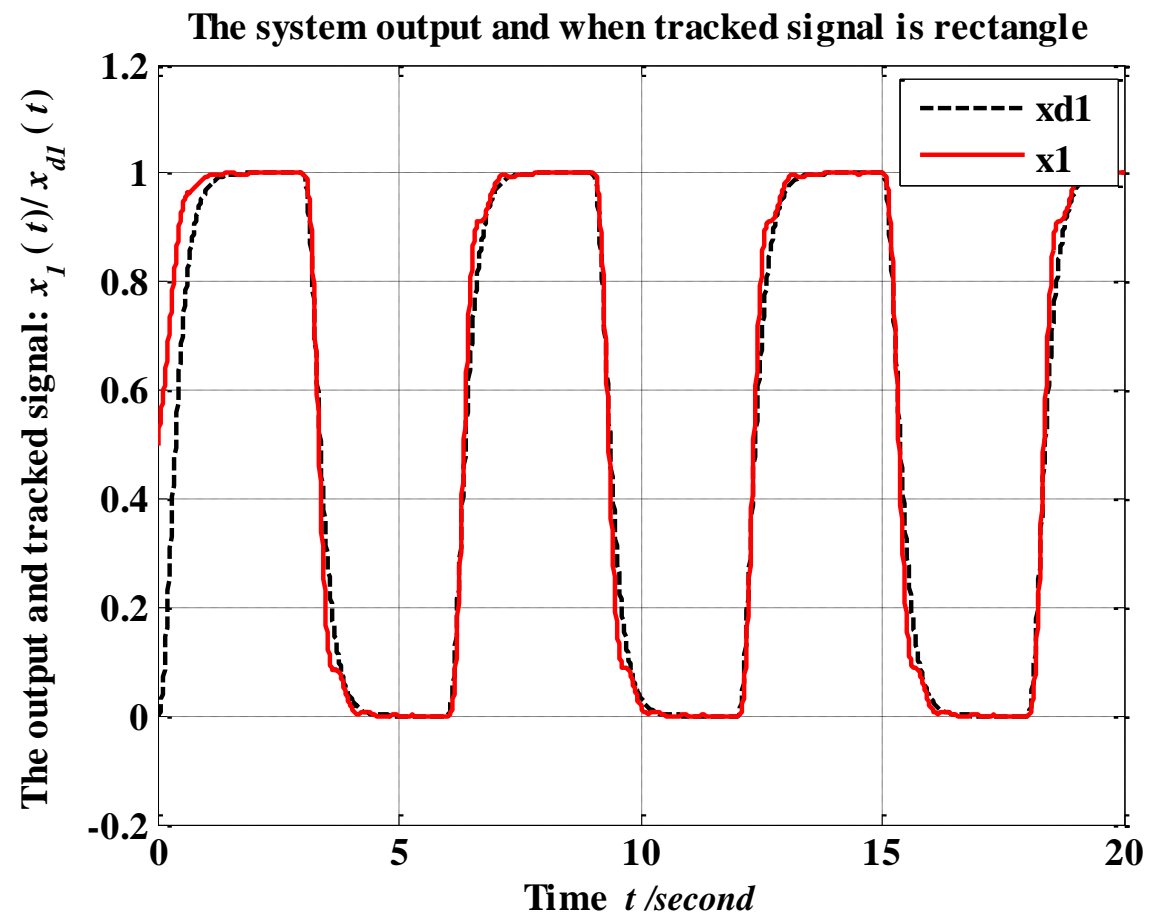

Figure 5. The system out response when the reference signal is rectangle
pulse. The parameter of robust observer $b_{2}=\left[\begin{array}{lll}16 & 0 & 0\end{array}\right]$

\section{Conclusions}

A kind of chattering free sliding mode control method by robust observer to design the switching control on the control derivative is presented. By the robust observer via LMI method, the immeasurable augmented state variables can be obtained for control systhesis even if there are unmodelled dynamics or disturbance on control gain. The derivative of the control signal is designed to form sliding mode control based on the robust observer. Therefore the chattering is eliminated completely in the method. The numerical simulation results prove its validity and effectiveness.

\section{References}

[1] D. Zhao, S. Li and Q. Zhu, "Output feedback terminal sliding mode control for a class of second order nonlinear systems", Asian Journal of Control, vol. 15, no. 237, (2013).

[2] Q. Ahmed, A. I.Bhatti and Q.Khan, "Condition monitoring of gasoline engine air intake system using second order sliding modes", International Journal of Vehicle Design, vol. 62, no.312, (2013).

[3] F. Nollet, T. Floquet and W. Perruquetti, "Observer-based second order sliding mode control laws for stepper motors", Control Engineering Practice, vol. 16, no. 429, (2008).

[4] N. K. M. Sirdi, A. Rabhi and L. Fridman, "Second order sliding-mode observer for estimation of vehicle dynamic parameters", International Journal of Vehicle Design, vol. 48, no. 190, (2008).

[5] G. Bartolini and P. Pydynowski, "Asymptotic linearization of uncertain nonlinear systems by means of continuous control”, International Journal of Robotic and Nonlinear Control, vol. 3, no. 87, (1993).

[6] G. Bartolini, and P. Pydynowski, "An improved, chattering free, V.S.C. scheme for uncertain dynamical system", IEEE, Transactions on Automatic Control, vol. 41, no. 1220, (1996).

[7] G. Bartolini, A. Ferrara and E. Usai, "Applications of a sub-optimal discontinuous control algorithm for uncertain second order systems", International Journal of Robotic and Nonlinear Control, vol. 7, no. 299, (1997). 
[8] G. Bartolini, A. Ferrara and E. Usai, "Chattering avoidance by second order sliding mode control", IEEE Transactions on Automatic Control, vol. 43, no. 241, (1998).

[9] G. Bartolini, A. Ferrara, E. Usai and Vadim I. Utkin, "On multi-input chattering-free second-order sliding mode control", IEEE, Transactions on Automatic Control, vol. 45, no. 1711, (2000).

[10] M. Ouassaid, M. Maaroufi and M. Cherkaoui, "Observer-based nonlinear control of power system using sliding mode control strategy", Electric Power Systems Research, vol. 84, no. 135, (2012).

[11] G. Wang, Z. Li and G. Zhang, "Quadrature PLL-based high-rrder sliding-mode observer for IPMSM sensorless control with online MTPA control strategy", IEEE, Transactions on Energy Conversion, vol. 28, no. 214, (2013).

[12] J. Hu and D. Zhu, "Vibration control of smart structure using sliding mode control with observer", Journal of Computers, vol.7, no. 411, (2012).

[13] L. Fridman, J. Davila and A. Levant, "High-order sliding-mode observation for linear systems with unknown inputs", Nonlinear analysis: Hybrid systems, vol. 5, no. 189, (2011).

[14] Y. B. Shtessel, S. Baev and C. Edwards, "HOSM observer for a class of non-minimum phase causal nonlinear MIMO systems", IEEE ,Transactions on Automatic Control, vol. 55, no. 543, (2010).

[15] F. J. Bejarano, L. M. Fridman and A. S. Poznyak, "Output integral sliding mode for min-max optimization of multi-plant linear uncertain systems", IEEE, Transactions on Automatic Control, vol. 54, no. 2611, (2010).

[16] T. M. M. Nasab, "A new approach for designing a PI controller via variable structure system theory", Proceedings of the 7th IEEE International Conference on Electronics, Circuits and Systems, Jounieh, Lebanon, (2000) 17-20 December.

[17] C. Edwards and S. K. Spurgeon, "On the development of discontinuous observers", International Journal of Control, vol. 59, no. 1211, (1994).

[18] C. Edwards and S. K. Spurgeon, "Use of nonlinear controller and observer theory for furnace emperature control", Proceedings of the International Conference on Control, Conventry, UK, (1994), 21-24 March.

[19] C. Edwards and S. K. Spurgeon, "On the limitations of some variable structure output feedback controller designs", Automatica, vol. 36, no. 743, (2000).

[20] M. Corless and J. Tu, "State and input estimation for a class of uncertain systems", Automatica, vol. 34, no. 757, (1998).

[21] J. C. Shen, B. S. Chen and F. C. Kung, "Memoryless stabilization of uncertain dynamic delay systems: Riccati equation approach", IEEE, Transactions on Automatic Control, vol. 36, no. 638, (1991).

Author

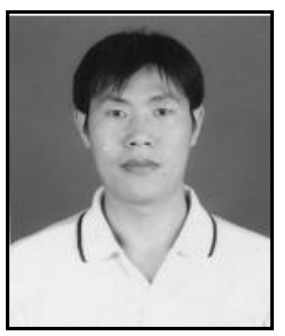

\section{Xiaoyu Zhang}

Dr. Xiaoyu Zhang was born in 1978. He received the B.S. degree from Yanshan University in 2000, the M.S. and Ph.D. degree both from Zhejiang University in 2003 and 2006 respectively. Now he is an Associate Professor of North China Institute of Science and Technology. His research interests include nonlinear control, intelligent control, switching systems and complex systems, etc. 